\title{
Design and Synthesis of New Amides and Thioamides Derived from 3,4-Ethylenedioxythiophene as Potential Anticonvulsants
}

\author{
Ravi Kulandasamy, Airody Vasudeva Adhikari, ${ }^{*}$ and James P. Stables ${ }^{\dagger}$ \\ Department of Chemistry, National Institute of Technology Karnataka, Surathkal, Srinivasnagar, Mangalore, \\ Karnataka 575 025, India.*E-mail: avchem@nitk.ac.in,avadhikari123@yahoo.co.in \\ ${ }^{\dagger}$ Preclinical Pharmacology Section, Epilepsy Branch, National Institute of Health, Bethesda, USA \\ Received May 10, 2010, Accepted September 15, 2010
}

\begin{abstract}
Five new series of 3,4-ethylenedioxythiophene derivatives carrying important pharamacophores, viz., amide, ester, ether and active secondary aryl moieties have been designed and synthesized through multistep reactions starting from thiodiglycolic ester and diethyl oxalate. They have been characterized by elemental and spectral data. All the target compounds have been screened for their anticonvulsant activity at three different models viz. maximal electroshock (MES), subcutaneous metrazole (scMET), and $6 \mathrm{~Hz}$ screen and evaluated for their neurotoxicity in rotorod model. Compound 6a emerged as lead with no neurotoxicity. All the five series of compounds are safe in the toxicity studies at the maximum dose of $300 \mathrm{mg} / \mathrm{kg}$ of body weight. Amongst the tested compounds, the ester pharmacophore with thioamide fragment has showed better activity than the other analogs.
\end{abstract}

Key Words: Thiophene, Thioamide, Amide, Anticonvulsant, Neurotoxicity

\section{Introduction}

One of the most common neurological disorders of the brain is epilepsy, which is due to a sudden burst of abnormal electrical discharges. The cumulated incidence of epilepsy, that is, the total number of persons who suffer multiple fits over an extended period of time, is about $5 \%$ among the developed countries. ${ }^{1}$ It has been reported that people suffering from epilepsy is increasing day by day in the world. Amongst the various active drugs available in the market, valpromide, phenacemide, levetiracetam, betamide, felbamate, paramethadione and gabapentin are noteworthy. Despite the optimal use of available antiepileptic drugs (AEDs), more than $20 \%$ of the world epileptic populations are not contented with the marketed AEDs. However, all currently approved anticonvulsant agents have dose-related toxicity and idiosyncratic side effects. ${ }^{2}$ Hence, a search for high activity with minimum toxicity is an area of investigation in medicinal chemistry.

Amide and its derivatives were shown to possess CNS activities such as antipsychotic, ${ }^{3}$ analgesic, ${ }^{4}$ anticonvulsant, ${ }^{5}$ antidepressant ${ }^{6}$ properties, etc. A thorough literature survey indicates that many anticonvulsants contain amide group as an important pharmacophore. As reported, many of the active compounds possess anilide ${ }^{7}$ and benzyl ${ }^{8}$ nucleus in their structures, as shown in Figure 1. It is well-documented that the combination of the above functional groups lead to potent anticonvulsant agents such as ameltolide, ${ }^{9}$ lacosamide, ${ }^{10}$ isoxazolecarboxmides, ${ }^{11}$ $N$-benzyl-2-(benzylamino)-4-hydroxybutanamide ${ }^{12}$ and functionalized amino ketones. ${ }^{13}$

Further, various benzothiazole derivatives were reported to possess good anticonvulsant property. The currently available active drug riluzole ${ }^{14}$ contains benzothiazole moiety as a scaffold in its structure. It was also evidenced that many benzothiazole derivatives carrying thiosemicarbazone ${ }^{15}$ and sulfonamide ${ }^{16}$ showed good protection against induced convulsion (Fig. 1).
Hence, benzothiazole moiety can be considered as one of the important pharmacophoric elements for the anticonvulsant activity.

A close observation of structures of the active drugs like stiripentol, ${ }^{17}$ topiramate, ${ }^{18}$ clearly reveals that the presence of dioxole group (Fig. 1) contributes to its activity to a larger extent. Further, it was shown that in many molecules the presence of cyclic ether ${ }^{19-21}$ functionality influenced the antiepileptic activity considerably. The observed results point out that cyclic ether functionality is one of the requirements to show good activity.

As reported in the literature, the presence of carboxylate group in 1-substituted-2-phenyl derivatives ${ }^{22}$ enhanced the anticonvulsant activity significantly. Further, Eddington ${ }^{23}$ et al. showed that enaminones (Fig. 1) containing ester pharmacophore exhibited better activity than the unsubstituted molecules, while Unverferth et al. evidenced that introduction of ester group in the pyrrole ${ }^{24}$ derivatives caused increased activity markedly.

In currently available active AEDs, like tiagabine ${ }^{25}$ and etizolam, ${ }^{26}$ the activity is mainly attributed to the presence of thiophene ring in their structures. Also, we reported ${ }^{27}$ that 3,4-disubstituted thiophenes, being hydrophobic moieties possess good anticonvulsant property with least neurotoxicity. Against this background, it was thought of designing new molecules containing hydrophobic thiophene moiety, active amide group, bioactive ester functionality and an electron releasing cyclic ether linkages as pharmacophoric elements. Also, it was contemplated to incorporate one more active amide in the new molecular framework in order to facilitate effective hydrogen bonding with receptor site and active distal ring like anilide, benzyl and benzothiazole moieties to provide them better binding ability. It was hoped that combination of these active pharmacophores in the new synthetic design would lead to better anticonvulsants with least neurotoxicity. The design of new target molecules has been summarized in the Figure 2 . 


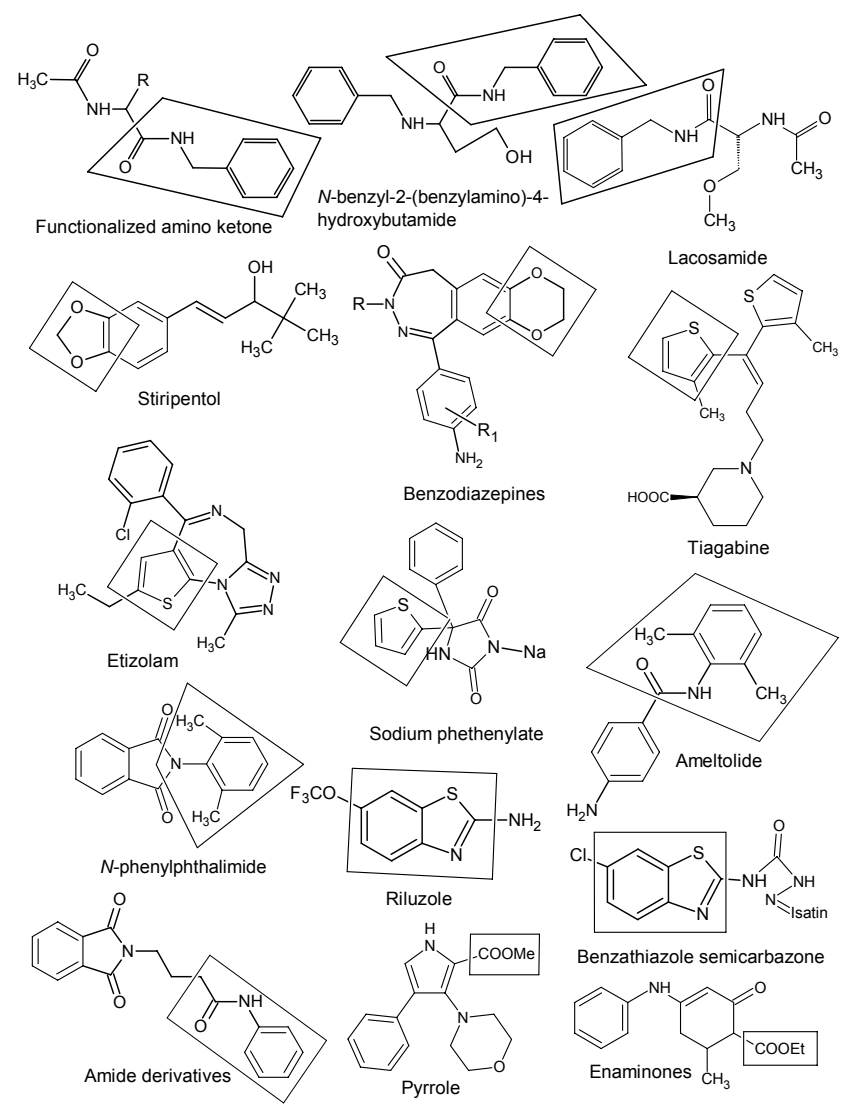

Figure 1. Structures of some important active agents and their important pharmacophores.

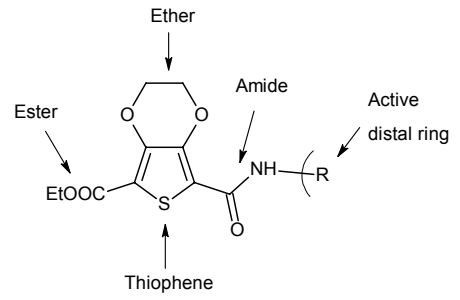

Figure 2. The structure of the newly designed molecule.

In the present paper, we report the synthesis and characterization of hitherto unknown 3,4-ethylenedioxy thiophene derivatives (4a-e, 6a-e, 9a-e, 11a-e and 15a-e). Also, we report their in vivo anticonvulsant activity by MES and scMET models and their neurotoxicity by rotorod model. Finally, we report the $6 \mathrm{~Hz}$ screening results of some of the selected compounds. Their structure-activity relationship was discussed.

\section{Experimental}

Chemistry. All the chemicals and the solvents, purchased from Aldrich and Merck were used without further purification. The progress of the reaction was monitored by thin layer chromatography, performed on a Silica gel $60 \mathrm{~F}_{254}$ coated Aluminium sheet. Melting points were determined on open capillaries using a Stuart SMP3 (BIBBY STERLIN Ltd. UK) apparatus and they were uncorrected. FTIR spectra were recorded on Nicolet Avatar 330 FTIR spectrophotometer. The ${ }^{1} \mathrm{H}$ NMR and ${ }^{13} \mathrm{C}$ NMR spectra were recorded on Varian 300 and $75 \mathrm{MHz}$ NMR spectrophotometers using TMS as an internal standard. Chemical shifts were reported in ppm $(\delta)$ and signals were described as singlet (s), doublet (d), triplet (t), quartet (q), broad (br) and multiplet (m). The coupling constant (J) values are expressed in Hz. The FAB mass spectra were recorded on a JEOL SX 102/DA-6000 spectrophotometer/Data system using Argon/ Xenon (6 KV, $10 \mathrm{~mA}) \mathrm{FAB}$ gas, at $70 \mathrm{eV}$. Elemental analysis was carried out using FLASH EA 1112 series, CHNSO Analyzer (Thermo). The compounds $\mathbf{1}$ and $\mathbf{7}$ were synthesized from the reported procedures. ${ }^{28}$

Synthesis of 7-(ethoxycarbonyl)-2,3-dihydrothieno[3,4-b] [1,4]dioxine-5-carboxylic acid (2): A mixture of diethyl-2,3dihydrothieno[3,4- $b][1,4]$ dioxine-5,7-dicarboxylate (1) (1 g, $0.0035 \mathrm{~mol}$ ) dissolved in $20 \mathrm{~mL}$ of absolute ethanol and $20 \mathrm{~mL}$ alcoholic sodium hydroxide $(0.0035 \mathrm{~mol})$ were refluxed for $5 \mathrm{~h}$. It was then concentrated to one third of its total volume under vacuum. The concentrate was diluted to $50 \mathrm{~mL}$ with water and stirred for $2 \mathrm{~h}$ at $60{ }^{\circ} \mathrm{C}$. The reaction mixture was cooled to 5 $10{ }^{\circ} \mathrm{C}$. The unreacted starting material that separated was removed by filtration. The filtrate was then acidified with conc. $\mathrm{HCl}$. The separated product was filtered and recrystallized using methanol to give 2 with yield, $78 \%$. mp 239 - $40{ }^{\circ} \mathrm{C}$. IR: (br) $2500-3000 \mathrm{~cm}^{-1}(-\mathrm{OH}), 1687 \mathrm{~cm}^{-1}(>\mathrm{C}=\mathrm{O}) ;{ }^{1} \mathrm{H}$ NMR (DMSO- $d_{6}$ $300 \mathrm{MHz}) \delta 1.26\left(\mathrm{t}, 3 \mathrm{H},-\mathrm{CH}_{3}\right.$-ester, $J=7.2$ ), 4.23 (q, $2 \mathrm{H}$, - $\mathrm{CH}_{2}$-ester, $J=7.2$ ), 4.34 (s, $4 \mathrm{H},-\mathrm{OCH}_{2} \mathrm{CH}_{2} \mathrm{O}-$ ), 13.27 [br, $1 \mathrm{H},-\mathrm{OH}\left(\mathrm{D}_{2} \mathrm{O}\right.$ exangeable)]; ${ }^{13} \mathrm{C}$ NMR (DMSO-d $\left.675 \mathrm{MHz}\right) \delta$ $14.0\left(-\mathrm{CH}_{3}\right), 60.8\left(-\mathrm{OCH}_{2}-\right), 65.9\left(-\mathrm{OCH}_{2}-\right), 110.0\left(\mathrm{C}_{3}\right.$ of thiophene), 112.1 ( $\mathrm{C}_{4}$ of thiophene $), 144.6\left(\mathrm{C}_{2}\right.$ of thiophene $), 145.1$ ( $\mathrm{C}_{5}$ of thiophene), 160.1 (-CO- of ester), 161.9 (-CO- acid); $\operatorname{MS}(\mathrm{m} / \mathrm{z}, \%): 258\left(\mathrm{M}^{+}, 70\right), 230(50), 213$ (100), 169 (40) and 142 (10).

General procedure for the synthesis of acid chloride (3) and (8). A mixture of $0.5 \mathrm{~g}$ of compound 2 or 7 and $15 \mathrm{~mL}$ of thionyl chloride were refluxed for $5 \mathrm{~h}$. Excess thionyl chloride was removed from the reaction mixture. The residual off-white solid was extracted with $50 \mathrm{~mL}$ of methylene dichloride and the organic layer was washed with saturated sodium bicarbonate solution $(25 \mathrm{~mL})$. After drying the organic layer with anhydrous sodium sulphate, the solvent was removed under vacuum to give the product, $\mathbf{3}$ or $\mathbf{8}$. This was used for next step without purification.

General procedure for synthesis of amides (4a-e) and (9a-e). To a clear solution of $0.5 \mathrm{~g}$ of acid chloride (3) or (8) in methylene dichloride $(20 \mathrm{~mL})$ containing $0.1 \mathrm{~mL}$ of pyridine as a catalyst, one/two molar quantity of respective amine were added with stirring. The stirring was continued for $12 \mathrm{~h}$. The reaction mass was then evaporated to dryness and diluted with $100 \mathrm{~mL}$ of water. The product was separated by filtration and recrystallized from appropriate solvent. The physical and characterization data of compounds, (4a-e) and (9a-e) are given in Table 1. The spectral data of some of the compounds are as follows.

Ethyl 7-[(2,6-dimethylphenyl)carbamoyl]-2,3-dihydrothieno [3,4-b][1,4]dioxine-5-carboxylate (4b): IR $\left(\mathrm{KBr}, \mathrm{cm}^{-1}\right)$ v 3367 (-NH-), 1720 (-CO- ester), 1655 (-CO- amide); ${ }^{1} \mathrm{H} \mathrm{NMR}\left(\mathrm{CDCl}_{3}\right.$, $300 \mathrm{MHz}) \delta 1.35\left(\mathrm{t}, 3 \mathrm{H},-\mathrm{CH}_{3}, J=6.9\right), 2.27$ (s, 6H, $-\mathrm{CH}_{3}$ phe- 
Table 1. Physical and characterization data of newly synthesized compounds

\begin{tabular}{|c|c|c|c|c|c|c|c|c|}
\hline \multirow{2}{*}{ Cpd } & \multirow{2}{*}{$\mathrm{R}$} & \multirow{2}{*}{ Mol. F/Mol. wt } & \multirow{2}{*}{$\begin{array}{c}\operatorname{mp}_{b}\left({ }^{\circ} \mathrm{C}\right) / \\
{ }^{\mathrm{Y}}(\%)\end{array}$} & \multirow{2}{*}{$\begin{array}{l}{ }^{a} \text { Recr. } \\
\text { Sol }\end{array}$} & \multicolumn{4}{|c|}{ Elemental analysis (\%): Found (cal.) } \\
\hline & & & & & $\mathrm{C}$ & $\mathrm{H}$ & $\mathrm{N}$ & $\mathrm{S}$ \\
\hline $4 a$ & aniline & $\mathrm{C}_{16} \mathrm{H}_{15} \mathrm{NO}_{5} \mathrm{~S} / 333$ & $161-62 / 72$ & $\mathrm{CHCl}_{3}$ & $57.65(57.71)$ & $4.54(4.62)$ & $4.20(4.29)$ & $9.62(9.51)$ \\
\hline $4 b$ & 2,6-xylidine & $\mathrm{C}_{18} \mathrm{H}_{19} \mathrm{NO}_{5} \mathrm{~S} / 361$ & $209-10 / 68$ & $\mathrm{MDC}$ & $59.82(59.78)$ & $5.30(5.42)$ & $3.88(3.79)$ & $8.87(8.92)$ \\
\hline $4 c$ & benzyl amine & $\mathrm{C}_{17} \mathrm{H}_{17} \mathrm{NO}_{5} \mathrm{~S} / 347$ & $123-24 / 80$ & $\mathrm{MeOH}$ & $58.78(58.86)$ & $4.93(4.99)$ & $4.03(4.08)$ & $9.23(9.16)$ \\
\hline $4 d$ & 2- $\mathrm{NH}_{2}$-benzothiazole & $\mathrm{C}_{17} \mathrm{H}_{14} \mathrm{~N}_{2} \mathrm{O}_{5} \mathrm{~S}_{2} / 390$ & $243-44 / 72$ & MDC & $52.30(52.41)$ & $3.61(3.68)$ & $7.17(7.26)$ & $16.43(16.49)$ \\
\hline $4 e$ & 4-NH${ }_{2}$-antipyrinyl & $\mathrm{C}_{21} \mathrm{H}_{21} \mathrm{~N}_{3} \mathrm{O}_{6} \mathrm{~S} / 443$ & $242-43 / 79$ & $\mathrm{MDC}$ & $56.87(56.95)$ & $4.77(4.85)$ & $9.48(9.55)$ & $7.23(7.15)$ \\
\hline $6 \mathbf{a}$ & aniline & $\mathrm{C}_{17} \mathrm{H}_{16} \mathrm{~N}_{2} \mathrm{O}_{5} \mathrm{~S}_{2} / 392$ & $256-57 / 57$ & DMSO & $52.03(52.09)$ & $4.11(4.16)$ & $7.14(7.19)$ & $16.34(16.26)$ \\
\hline $6 b$ & 2,6-xylidine & $\mathrm{C}_{19} \mathrm{H}_{20} \mathrm{~N}_{2} \mathrm{O}_{5} \mathrm{~S}_{2} / 420$ & $201-02 / 72$ & DMF & $54.27(54.35)$ & $4.79(4.86)$ & $6.66(6.75)$ & $15.25(15.17)$ \\
\hline $6 c$ & benzyl amine & $\mathrm{C}_{18} \mathrm{H}_{18} \mathrm{~N}_{2} \mathrm{O}_{5} \mathrm{~S}_{2} / 406$ & $217-18 / 68$ & DMF & $53.19(53.26)$ & $4.46(4.54)$ & $6.89(6.95)$ & $15.78(15.69)$ \\
\hline $6 d$ & 2- $\mathrm{NH}_{2}$-benzothiazole & $\mathrm{C}_{18} \mathrm{H}_{15} \mathrm{~N}_{3} \mathrm{O}_{5} \mathrm{~S}_{3} / 449$ & $243-44 / 60$ & DMF & $48.09(48.16)$ & $3.36(3.43)$ & $9.35(9.43)$ & $21.40(21.32)$ \\
\hline $6 e$ & 4- $\mathrm{NH}_{2}$-antipyrinyl & $\mathrm{C}_{22} \mathrm{H}_{22} \mathrm{~N}_{4} \mathrm{O}_{6} \mathrm{~S}_{2} / 502$ & $235-36 / 69$ & DMF & $52.58(52.65)$ & $4.41(4.35)$ & $11.15(11.21)$ & $12.76(12.69)$ \\
\hline $9 \mathbf{a}$ & aniline & $\mathrm{C}_{20} \mathrm{H}_{16} \mathrm{~N}_{2} \mathrm{O}_{4} \mathrm{~S} / 380$ & $>300 / 72$ & DMF & $63.14(63.19)$ & $4.24(4.29)$ & $7.36(7.39)$ & $8.43(8.39)$ \\
\hline $9 \mathrm{~b}$ & 2,6-xylidine & $\mathrm{C}_{24} \mathrm{H}_{24} \mathrm{~N}_{2} \mathrm{O}_{4} \mathrm{~S} / 436$ & $>300 / 75$ & DMF & $66.03(66.12)$ & $5.54(5.56)$ & $6.42(6.45)$ & $7.35(7.33)$ \\
\hline $9 c$ & benzyl amine & $\mathrm{C}_{22} \mathrm{H}_{20} \mathrm{~N}_{2} \mathrm{O}_{4} \mathrm{~S} / 408$ & $232-33 / 65$ & DMF & $64.69(64.75)$ & $4.94(4.99)$ & $6.86(6.94)$ & $7.85(7.78)$ \\
\hline 9d & 2- $\mathrm{NH}_{2}$-benzothiazole & $\mathrm{C}_{22} \mathrm{H}_{14} \mathrm{~N}_{4} \mathrm{O}_{4} \mathrm{~S}_{3} / 494$ & $>300 / 72$ & DMF & $53.43(53.49)$ & $2.85(2.83)$ & $11.33(11.40)$ & 19.45 (19.38) \\
\hline $9 e$ & 4- $\mathrm{NH}_{2}$-antipyrinyl & $\mathrm{C}_{30} \mathrm{H}_{28} \mathrm{~N}_{6} \mathrm{O}_{6} \mathrm{~S} / 600$ & $283-84 / 70$ & DMF & $59.99(60.07)$ & $4.70(4.65)$ & $13.99(14.05)$ & $5.34(5.29)$ \\
\hline 11a & aniline & $\mathrm{C}_{22} \mathrm{H}_{18} \mathrm{~N}_{4} \mathrm{O}_{4} \mathrm{~S}_{3} / 498$ & $>300 / 60$ & DMF & $53.00(53.11)$ & $3.64(3.58)$ & $11.24(11.29)$ & $19.29(19.20)$ \\
\hline $11 b$ & 2,6-xylidine & $\mathrm{C}_{26} \mathrm{H}_{26} \mathrm{~N}_{4} \mathrm{O}_{4} \mathrm{~S}_{3} / 554$ & $>300 / 74$ & DMF & $56.30(56.37)$ & $4.72(4.79)$ & $10.10(10.16)$ & $17.34(17.31)$ \\
\hline $11 \mathrm{c}$ & benzyl amine & $\mathrm{C}_{24} \mathrm{H}_{22} \mathrm{~N}_{4} \mathrm{O}_{4} \mathrm{~S}_{3} / 526$ & $265-66 / 67$ & DMF & $54.73(54.77)$ & $4.21(4.26)$ & $10.64(10.57)$ & $18.27(18.35)$ \\
\hline $11 d$ & 2-NH -benzothiazole & $\mathrm{C}_{24} \mathrm{H}_{16} \mathrm{~N}_{6} \mathrm{O}_{4} \mathrm{~S}_{5} / 612$ & $250-51 / 63$ & DMF & $47.04(47.09)$ & $2.63(2.59)$ & $13.72(13.79)$ & $26.16(26.19)$ \\
\hline $11 \mathrm{e}$ & 4- $\mathrm{NH}_{2}$-antipyrinyl & $\mathrm{C}_{32} \mathrm{H}_{30} \mathrm{~N}_{8} \mathrm{O}_{6} \mathrm{~S}_{3} / 718$ & $189-90 / 74$ & $\mathrm{CHCl}_{3}$ & $53.47(53.57)$ & $4.21(4.29)$ & $15.59(15.67)$ & $13.38(13.26)$ \\
\hline \multirow{2}{*}{ Cpd } & \multirow{2}{*}{$\mathrm{R}_{1}$} & \multirow{2}{*}{ Mol. F/Mol. wt } & \multirow{2}{*}{$\begin{array}{c}\operatorname{mp}_{b}\left({ }^{\circ} \mathrm{C}\right) / \\
{ }^{\mathrm{Y}}(\%)\end{array}$} & \multirow{2}{*}{$\begin{array}{c}{ }^{a} \text { Recr. } \\
\text { Sol }\end{array}$} & \multicolumn{4}{|c|}{ Elemental analysis (\%): Found (cal.) } \\
\hline & & & & & $\mathrm{C}$ & $\mathrm{H}$ & $\mathrm{N}$ & $\mathrm{S}$ \\
\hline $15 \mathbf{a}$ & aniline & $\mathrm{C}_{20} \mathrm{H}_{15} \mathrm{NO}_{4} \mathrm{~S} / 365$ & $190-91 / 78$ & $\mathrm{CHCl}_{3}$ & $65.74(65.79)$ & $4.14(4.19)$ & $3.83(3.89)$ & $8.78(8.72)$ \\
\hline $15 b$ & 2,6-xylidine & $\mathrm{C}_{22} \mathrm{H}_{19} \mathrm{NO}_{4} \mathrm{~S} / 393$ & $241-42 / 55$ & $\mathrm{MDC}$ & $67.16(67.25)$ & $4.87(4.95)$ & $3.56(3.64)$ & $8.15(8.19)$ \\
\hline $15 c$ & benzyl amine & $\mathrm{C}_{21} \mathrm{H}_{17} \mathrm{NO}_{4} \mathrm{~S} / 379$ & $226-27 / 72$ & $\mathrm{MDC}$ & $66.47(66.56)$ & $4.52(4.59)$ & $3.69(3.76)$ & $8.45(8.54)$ \\
\hline $15 d$ & 2- $\mathrm{NH}_{2}$-benzothiazole & $\mathrm{C}_{21} \mathrm{H}_{14} \mathrm{~N}_{2} \mathrm{O}_{4} \mathrm{~S}_{2} / 422$ & $268-69 / 63$ & $\mathrm{MDC}$ & $59.70(59.76)$ & $3.34(3.39)$ & $6.63(6.72)$ & $15.18(15.26)$ \\
\hline $15 e$ & 4- $\mathrm{NH}_{2}$-antipyrinyl & $\mathrm{C}_{25} \mathrm{H}_{21} \mathrm{~N}_{3} \mathrm{O}_{5} \mathrm{~S} / 475$ & $256-57 / 76$ & $\mathrm{MDC}$ & $63.15(63.24)$ & $4.45(4.52)$ & $8.84(8.93)$ & $6.74(6.77)$ \\
\hline
\end{tabular}

*Recrystallization solvent, ${ }^{\#}$ Yield after recrystallization.

nyl), 4.32 (q, 2H, - $\left.\mathrm{OCH}_{2-}, J=6.9\right), 4.44\left(\mathrm{~s}, 4 \mathrm{H},-\mathrm{OCH}_{2}-\right), 7.1$ (s, 3H, phenyl), 8.15 (s, 1H, -NH-); ${ }^{13} \mathrm{C} \mathrm{NMR}\left(\mathrm{CDCl}_{3}, 75 \mathrm{MHz}\right)$ $\delta 14.2\left(-\mathrm{CH}_{3}\right.$ of ester), 18.5 (- $\mathrm{CH}_{3}$ of aromatic), $61.1\left(-\mathrm{OCH}_{2}-\right)$, 64.6-65.2 $\left(-\mathrm{OCH}_{2} \mathrm{CH}_{2} \mathrm{O}-\right), 111.4\left(\mathrm{C}_{3}\right.$ of thiophene $), 118.5\left(\mathrm{C}_{1}\right.$ of phenyl), $127.3\left(\mathrm{C}_{3} \& \mathrm{C}_{5}\right.$ of phenyl), $128.1\left(\mathrm{C}_{2} \& \mathrm{C}_{6}\right.$ of phenyl), $133.3\left(\mathrm{C}_{4}\right.$ of thiophene), $135.2\left(\mathrm{C}_{4}\right.$ of phenyl $), 140.0\left(\mathrm{C}_{2}\right.$ of thiophene), 144.4 ( $\mathrm{C}_{5}$ of thiophene), 158.4 (-CONH-), 160.8 (-CO- of ester); MS (FAB) ( $m / z, \%): 361(\mathrm{M}+1,100), 317(70)$, 241 (80), 213 (50), 169 (50).

Ethyl 7-(benzylcarbamoyl)-2,3-dihydrothieno[3,4-b][1,4] dioxine-5-carboxylate (4c): IR ( $\left.\mathrm{KBr}, \mathrm{cm}^{-1}\right)$ v 3396 (-NH-), 1712 (-CO- ester), 1676 (-CO- amide); ${ }^{1} \mathrm{H}$ NMR $\left(\mathrm{CDCl}_{3}, 300 \mathrm{MHz}\right)$ $\delta 1.32\left(\mathrm{t}, 3 \mathrm{H},-\mathrm{CH}_{3}, J=6.9\right), 3.30\left(\mathrm{~s}, 2 \mathrm{H},-\mathrm{NCH}_{2}-\right), 4.28(\mathrm{q}, 2 \mathrm{H}$, $\left.-\mathrm{OCH}_{2-}, J=6.9\right), 4.35$ (d, 2H, $\left.-\mathrm{OCH}_{2^{-}}, J=6.3\right), 4.56(\mathrm{~d}, 2 \mathrm{H}$, $\left.-\mathrm{OCH}_{2-}, J=6\right), 7.28\left(\mathrm{~m}, 5 \mathrm{H}\right.$, phenyl), $8.00(\mathrm{~s}, 1 \mathrm{H},-\mathrm{NH}-) ;{ }^{13} \mathrm{C}$ $\mathrm{NMR}\left(\mathrm{CDCl}_{3}, 75 \mathrm{MHz}\right) \delta 14.5\left(-\mathrm{CH}_{3}\right), 44.4\left(-\mathrm{NCH}_{2}-\right), 62.2$ $\left(-\mathrm{OCH}_{2}\right), 66.0-66.5\left(-\mathrm{OCH}_{2} \mathrm{CH}_{2} \mathrm{O}-\right), 118.6\left(\mathrm{C}_{3}\right.$ of thiophene $)$, $128.3\left(\mathrm{C}_{4}\right.$ of thiophene $), 128.5\left(\mathrm{C}_{2} \& \mathrm{C}_{6}\right.$ of phenyl $), 129.6\left(\mathrm{C}_{3}\right.$, $\mathrm{C}_{4} \& \mathrm{C}_{5}$ of thiophene), 139.8 ( $\mathrm{C}_{1}$ of phenyl), $142.4\left(\mathrm{C}_{2}\right.$ of thiophene), 146.5 ( $\mathrm{C}_{5}$ of thiophene), 162.8 (-CO- of ester); MS (FAB) $(\mathrm{m} / \mathrm{z}, \%): 348(\mathrm{M}+1,100), 302(10), 273(10), 241(30)$, $213(10)$.

Ethyl 7-[(1,5-dimethyl-3-oxo-2-phenyl-2,3-dihydro-1 $H$-pyrazol-4-yl)carbamoyl]-2,3-dihydrothieno[3,4-b][1,4]dioxine- 5-carboxylate (4e): IR (KBr, cm $\left.{ }^{-1}\right)$ v 3207 (-NH-), 1669 (-CO-

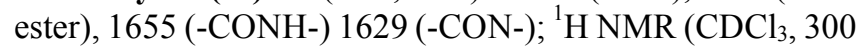
$\mathrm{MHz}) \delta 1.28\left(\mathrm{t}, 3 \mathrm{H},-\mathrm{CH}_{3}, J=6.9\right), 2.27\left(\mathrm{~s}, 3 \mathrm{H},-\mathrm{CH}_{3}\right.$ antipyrine), 3.03 (s, 3H, $-\mathrm{NCH}_{3}$ ), 4.27 (q, $\left.2 \mathrm{H},-\mathrm{OCH}_{2-}, J=7.2\right), 4.35$ (s, 4H, $\left.-\mathrm{OCH}_{2}-\right), 7.21-7.42$ (m, 5H, aromatic), 8.18 (s, $\left.1 \mathrm{H},-\mathrm{NH}-\right) ;{ }^{13} \mathrm{C}$ $\mathrm{NMR}\left(\mathrm{CDCl}_{3}, 75 \mathrm{MHz}\right) \delta 12.6\left(-\mathrm{CH}_{3}\right), 14.1\left(-\mathrm{CH}_{3}\right.$ ester $), 36.0$ $\left(-\mathrm{NCH}_{2}-\right), 61.1\left(-\mathrm{OCH}_{2}-\right), 64.6-65.1\left(-\mathrm{OCH}_{2} \mathrm{CH}_{2} \mathrm{O}-\right), 108.0\left(\mathrm{C}_{3}\right.$ of pyrazolinone), $111.6\left(\mathrm{C}_{4}\right.$ of thiophene $), 117.7\left(\mathrm{C}_{3}\right.$ of thiophene), 124.0 ( $\mathrm{C}_{2} \& \mathrm{C}_{6}$ of phenyl), 126.8 ( $\mathrm{C}_{1}$ of phenyl), 129.1 $\left(\mathrm{C}_{3} \& \mathrm{C}_{5}\right.$ of phenyl), 134.5 ( $\mathrm{C}_{4}$ of phenyl), $140.4\left(\mathrm{C}_{5}\right.$ of thiophene), 144.4 ( $\mathrm{C}_{2}$ of thiophene), 149.0 ( $\mathrm{C}_{1}$ of pyrazolinone), 158.9 (-CO- of pyrazolinone), 160.7 (-CO- ester), 161.3 (-CONH-); MS (FAB) $(m / z, \%): 444$ (M+1, 100), 398 (10), 371 (10), 213 (30), 241 (100), 230 (10), 169 (30).

$N, N$-Diphenyl-2,3-dihydrothieno[3,4- $b][1,4]$ dioxine-5,7dicarboxamide (9a): IR (KBr, cm ${ }^{-1}$ ) v 3381 (-NH-), 1666 (-COamide); ${ }^{1} \mathrm{H}$ NMR $\left(\mathrm{CDCl}_{3}, 300 \mathrm{MHz}\right) \delta 4.59\left(\mathrm{~s}, 4 \mathrm{H},-\mathrm{OCH}_{2}\right.$ $\mathrm{CH}_{2} \mathrm{O}-$ ), 7.16 (s, 2H, C 4 of phenyl), 7.39 (s, 4H, $\mathrm{C}_{3} \& \mathrm{C}_{5}$ of phenyl), 7.69 (s, 4H, $\mathrm{C}_{2} \& \mathrm{C}_{6}$ of phenyl), 9.24 (s, 2H, -NH-); MS (FAB) $(m / z, \%): 381(\mathrm{M}+1,100), 349(5), 303$ (10), 288 (80), 261 (10), $226(5), 121$ (10).

$\boldsymbol{N}, \boldsymbol{N}$-2,6-Dimethylphenyl-2,3-dihydrothieno $[3,4-b][1,4]$ dioxine-5,7-dicarboxamide (9b): IR ( $\left.\mathrm{KBr}, \mathrm{cm}^{-1}\right)$ v 3371 (-NH-), 1654 (-CO- amide); ${ }^{1} \mathrm{H} \mathrm{NMR}\left(\mathrm{CDCl}_{3}, 300 \mathrm{MHz}\right) \delta 2.28(\mathrm{~s}, 12 \mathrm{H}$, 
$\left.-\mathrm{CH}_{3}\right), 4.59\left(\mathrm{~s}, 4 \mathrm{H},-\mathrm{OCH}_{2}-\right), 7.12(\mathrm{~s}, 6 \mathrm{H}$, phenyl), $8.20(\mathrm{~s}, 1 \mathrm{H}$, -NH-); MS (FAB) ( $m / z, \%): 437$ (M+1, 80), 424 (10), $316(10)$, 289 (50), 120 (30).

General procedure for the preparation of thioamides (6a-e) and 11 (a-e): To a clear solution of $0.5 \mathrm{~g}(0.0018 \mathrm{~mol})$ of acid chloride (3) in $25 \mathrm{~mL}$ of methylene dichloride, ammonium thiocyanate powder $(0.0027 \mathrm{~mol})$ and polyethylene glycol-600 $(0.1 \mathrm{~g})$ as PTC were added. After stirring the reaction mixture for $2 \mathrm{~h}$ at $25^{\circ} \mathrm{C}$ the corresponding amine $(0.0018 \mathrm{~mol})$ was added and the reaction mixture was further stirred for $4 \mathrm{~h}$ at room temperature. Then, $25 \mathrm{~mL}$ of water was added to the well-stirred mixture and the resulting slurry was filtered, washed with water, dried and finally recrystallized from appropriate solvent to obtain the title compounds (6a-e). The compounds 11a-e were obtained by using ammonium thiocyanate $(0.0054 \mathrm{~mol})$ and two moles of corresponding amine. The physical and characterization data of compounds, (6a-e) and (11a-e) are tabulated in Table 1. The spectral data of some of the compounds are given below:

Ethyl 7-[(phenylcarbamothioyl)carbamoyl]-2,3-dihydrothieno $[3,4-b][1,4]$ dioxine-5-carboxylate (6a): $\mathrm{IR}\left(\mathrm{KBr}, \mathrm{cm}^{-1}\right)$ v 3337 (-NH-), 1719 (-CO- ester), 1695 (-CONH-), 1153 (-CS-); MS (FAB) (m/z, \%): 393 (M+1, 50), 360 (5), 307 (40), 289 (20), 241 (60), 213 (10), 167 (20).

Ethyl 7-\{[(2,6-dimethylphenyl)carbamothioyl]carbamoyl $\}$ 2,3-dihydrothieno[3,4-b][1,4] dioxine-5-carboxylate $(6 \mathrm{~b})$ : IR $\left(\mathrm{KBr}, \mathrm{cm}^{-1}\right)$ v 3365 (-NH-), 1716 (-CO- ester), 1668 (-CONH-), 1159 (-CS-); ${ }^{1} \mathrm{H} \mathrm{NMR}\left(\mathrm{CDCl}_{3}, 300 \mathrm{MHz}\right) \delta 1.38\left(\mathrm{t}, 3 \mathrm{H},-\mathrm{CH}_{3}\right.$, $J=6.9), 2.30$ (s, 6H, $-\mathrm{CH}_{3}$ aromatic), 4.37 (q, $2 \mathrm{H},-\mathrm{CH}_{2-}, J=$ 6.9), 4.46 (s, $\left.2 \mathrm{H},-\mathrm{OCH}_{2}-\right), 4.55\left(\mathrm{~s}, 2 \mathrm{H},-\mathrm{OCH}_{2}-\right), 7.12-7.22(\mathrm{~m}$, $3 \mathrm{H}$, phenyl), 9.96 (s, 1H, -NHCS-), 11.57 (s, 1H, -NHCO-); ${ }^{13} \mathrm{C} \mathrm{NMR}\left(\mathrm{CDCl}_{3}, 75 \mathrm{MHz}\right) \delta 14.1\left(-\mathrm{CH}_{3}\right), 18.1\left(-\mathrm{CH}_{3}\right), 61.6$ $\left(-\mathrm{OCH}_{2}-\right), 64.5\left(-\mathrm{OCH}_{2}-\right), 65.7\left(-\mathrm{OCH}_{2}-\right), 114.8\left(\mathrm{C}_{3}\right.$ of thiophene $), 114.9$ ( $\mathrm{C}_{1}$ of phenyl), $128.2\left(\mathrm{C}_{2} \& \mathrm{C}_{6}\right.$ of phenyl), 135.2 $\left(\mathrm{C}_{4}\right.$ of thiophene), $135.4\left(\mathrm{C}_{3}, \mathrm{C}_{4} \& \mathrm{C}_{5}\right.$-phenyl $), 142.0\left(\mathrm{C}_{2}\right.$ of thiophene), 144.1 ( $\mathrm{C}_{5}$ of thiophene), 159.8 (-CONH-), 160.2 (-CO- ester), 179.4 (-CSNH-); MS (FAB) ( $\mathrm{m} / \mathrm{z}, \%): 421$ (M+1, 100), 405 (10), 317 (10), 258 (50), 241 (80), 213 (50), 207 (10), 181 (5), 169 (20).

Ethyl 7-[(benzylcarbamothioyl)carbamoyl]-2,3-dihydrothieno $[3,4-b][1,4]$ dioxine-5-carboxylate (6c): $\mathrm{IR}\left(\mathrm{KBr}, \mathrm{cm}^{-1}\right)$ v 3348 (-NH-), 1714 (-CO- ester), 1655 (-CONH-), 1159 (-CS-); ${ }^{1} \mathrm{H}$ NMR $\left(\mathrm{CDCl}_{3}, 300 \mathrm{MHz}\right) \delta 1.36\left(\mathrm{t}, 3 \mathrm{H},-\mathrm{CH}_{3}, J=7.2\right), 4.34$ (q, $\left.2 \mathrm{H},-\mathrm{OCH}_{2^{-}}, J=7.2\right), 4.46\left(\mathrm{~s}, 2 \mathrm{H},-\mathrm{OCH}_{2}-\right), 4.53(\mathrm{~s}, 2 \mathrm{H}$, $\left.-\mathrm{OCH}_{2}-\right), 4.88\left(\mathrm{~d}, 2 \mathrm{H},-\mathrm{NHCH}_{2}-, J=5.1\right), 7.33$ (m, 5H, phenyl), 9.77 (s, $1 \mathrm{H},-\mathrm{NHCO}-), 10.74$ (s, 1H, -NHCS-); ${ }^{13} \mathrm{C} \mathrm{NMR}\left(\mathrm{CDCl}_{3}\right.$, $75 \mathrm{MHz}) \delta 14.1\left(-\mathrm{CH}_{3}\right), 49.7\left(-\mathrm{NHCH}_{2}-\right), 61.6\left(-\mathrm{OCH}_{2}-\right), 64.5-$ $65.7\left(-\mathrm{OCH}_{2} \mathrm{CH}_{2} \mathrm{O}-\right), 114.6\left(\mathrm{C}_{3}\right.$ of thiophene $), 115.0\left(\mathrm{C}_{1}\right.$ of phenyl), 127.8 ( $\mathrm{C}_{4}$ of thiophene $), 127.9\left(\mathrm{C}_{2} \& \mathrm{C}_{6}\right.$ of phenyl), $128.8\left(\mathrm{C}_{3} \& \mathrm{C}_{4}\right.$ of phenyl), $136.0\left(\mathrm{C}_{4}\right.$ of phenyl), $141.8\left(\mathrm{C}_{5}\right.$ of thiophene), 144.1 ( $\mathrm{C}_{2}$ of thiophene), 159.5 (-CONH-), 160.2 (-CO- ester), 179.5 (-CSNH-); MS (FAB) ( $\mathrm{m} / \mathrm{z}, \%): 407$ (M+1, 100), 325 (40), 295 (5), 281 (80), 267 (50), 258 (30), 241 (80), 194 (10).

Ethyl 7-[(1,3-benzothiazol-2-ylcarbamothioyl)carbamoyl]2,3-dihydrothieno[3,4-b][1,4] dioxine-5-carboxylate (6d): IR $\left(\mathrm{KBr}, \mathrm{cm}^{-1}\right)$ v 3329 (-NH-), 1693 (-CO- ester), 1658 (-CONH-), 1153 (-CS-); ${ }^{1} \mathrm{H} \mathrm{NMR}\left(\mathrm{CDCl}_{3}, 300 \mathrm{MHz}\right) \delta 1.39\left(\mathrm{t}, 3 \mathrm{H},-\mathrm{CH}_{3}\right.$,
$J=6.9), 4.37$ (q, 2H, $\left.-\mathrm{OCH}_{2-}, J=6.9\right), 4.52$ (d, 2H, $-\mathrm{CH}_{2} \mathrm{O}-$, $J=3.9)$, 4.63 (d, 2H, $\left.-\mathrm{OCH}_{2-}, J=3.9\right)$, 7.37-7.51 (m, 4H, phenyl), 10.03 (s, 1H, -NHCS-), 11.57 (s, 1H, -NHCO-); MS (FAB) (m/z, \%): $450(\mathrm{M}+1,40), 419(50), 391$ (100), 317 (80), 307 (30), 258 (10), 241 (100), 213 (60), 193 (10).

$N, N$-Bis(2,6-dimethylphenylcarbamothioyl)-2,3-dihydrothieno[3,4-b][1,4]dioxine-5,7-dicarboxamide (11b): IR (KBr, $\mathrm{cm}^{-1}$ ) v 3359 (-NH-), 1661 (-CONH-), 1150 (-CS-); ${ }^{1} \mathrm{H}$ NMR $\left(\mathrm{CDCl}_{3}, 300 \mathrm{MHz}\right) \delta 2.30\left(\mathrm{~s}, 12 \mathrm{H},-\mathrm{CH}_{3}\right), 4.60\left(\mathrm{~s}, 4 \mathrm{H},-\mathrm{OCH}_{2}\right.$ $\mathrm{CH}_{2} \mathrm{O}-$ ), 7.20 (m, 6H, phenyl), 9.90 (s, 2H, -NHCO-), 11.50 (s, 2H, -NHCS-); ${ }^{13} \mathrm{C} \mathrm{NMR}\left(\mathrm{CDCl}_{3}, 75 \mathrm{MHz}\right) \delta 18.1\left(-\mathrm{CH}_{3}\right)$, $65.7\left(-\mathrm{OCH}_{2}-\right), 70.4\left(-\mathrm{OCH}_{2}-\right), 117.9\left(\mathrm{C}_{1}\right.$ of phenyl $), 128.3\left(\mathrm{C}_{2}\right.$ \& $\mathrm{C}_{6}$ of phenyl), $135.1\left(\mathrm{C}_{3} \& \mathrm{C}_{4}\right.$ of thiophene $), 135.3\left(\mathrm{C}_{3}, \mathrm{C}_{4} \&\right.$ $\mathrm{C}_{5}$ of phenyl), 141.2 ( $\mathrm{C}_{2} \& \mathrm{C}_{5}$ of thiophene), 159.2 (-CONH-), 179.2 (-CSNH-); MS (FAB) $(\mathrm{m} / \mathrm{z}, \%): 555(\mathrm{M}+1,100), 435$ (60), 391 (20), $376(20), 348$ (70), 272 (20), 228 (50), 212 (80), 169 (70).

$N, N$-Bis(benzylcarbamothioyl)-2,3-dihydrothieno [3,4-b] [1,4]dioxine-5,7-dicarboxamide (11c): IR $\left(\mathrm{KBr}, \mathrm{cm}^{-1}\right)$ v 3344

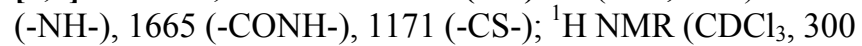
$\mathrm{MHz}) \delta 3.51\left(\mathrm{~s}, 4 \mathrm{H},-\mathrm{NCH}_{2}-\right), 4.67\left(\mathrm{~s}, 4 \mathrm{H},-\mathrm{OCH}_{2}-\right), 7.27-7.37$ (m, 10H, phenyl), 10.73 (s, 2H, -NHCO-); MS (FAB) ( $/ \mathrm{z}, \%$ ): 526 (30), 437 (20), 421 (10), 329 (5), 273 (10), 165 (10), 107 (20).

$\boldsymbol{N}, \boldsymbol{N}$-Bis(1,5-dimethyl-3-oxo-2-phenyl-2,3-dihydro-1 $H$-pyrazol-4-ylcarbamoyl)-2,3-dihydro-thieno[3,4-b][1,4]dioxine5,7-dicarboxamide (11e): IR (KBr, cm $\left.{ }^{-1}\right)$ v 3344 (-NH-), 1664 (-CONH-), 1143 (-CS-); ${ }^{1} \mathrm{H}$ NMR (CDCl $\left.3,300 \mathrm{MHz}\right) \delta 2.27$ (s, $6 \mathrm{H},-\mathrm{CH}_{3}$ of antipyrene), $3.15\left(\mathrm{~s}, 6 \mathrm{H},-\mathrm{NCH}_{3}\right), 4.55(\mathrm{~s}, 4 \mathrm{H}$, $-\mathrm{OCH}_{2}-$ ), 7.28-7.46 (m, 10H, aromatic), 9.88 (s, 2H, -CONH-), 11.18 (s, 2H, -CSNH-); ${ }^{13} \mathrm{C} \mathrm{NMR}\left(\mathrm{CDCl}_{3}, 75 \mathrm{MHz}\right) \delta 11.9$ $\left(-\mathrm{CH}_{3}\right), 35.6\left(-\mathrm{NCH}_{3}\right), 69.9-70.3\left(-\mathrm{OCH}_{2} \mathrm{CH}_{2} \mathrm{O}-\right), 108.9\left(\mathrm{C}_{2}\right.$ of pyrazolinone), $117.6\left(\mathrm{C}_{3} \& \mathrm{C}_{4}\right.$ of thiophene $), 124.4\left(\mathrm{C}_{2} \& \mathrm{C}_{6}\right.$ of phenyl), 127.0 ( $\mathrm{C}_{1}$ of phenyl), $129.1\left(\mathrm{C}_{3} \& \mathrm{C}_{5}\right.$ of phenyl), 134.5 ( $\mathrm{C}_{4}$ of phenyl), $141.3\left(\mathrm{C}_{2} \& \mathrm{C}_{5}\right.$ of thiophene), $151.7\left(\mathrm{C}_{1}\right.$ of pyrazolinone), 158.8 (-CO- of pyrazolinone), 161.1 (-CONH-), 180.6 (-CSNH-).

Synthesis of 7-(phenylcarbonyl)-2,3-dihydrothieno[3,4-b] [1,4]dioxine-5-carboxylic acid (13): A clear solution of $1 \mathrm{~g}$ $(0.0036 \mathrm{~mol})$ of acid chloride 3 in $50 \mathrm{~mL}$ of methylene dichloride were added into $50 \mathrm{~mL}$ of benzene containing anhydrous aluminium chloride $1.22 \mathrm{~g}(0.009 \mathrm{~mol})$ at $10-15^{\circ} \mathrm{C}$ for about $30 \mathrm{~min}$. After completion of the addition, the reaction mixture was allowed to stir at $25^{\circ} \mathrm{C}$ for $3 \mathrm{~h}$. Then, $50 \mathrm{~mL}$ of cold $5 \%$ $\mathrm{HCl}$ was added into the reaction mixture and stirred for $10 \mathrm{~min}$. Organic layer was separated and extracted using $2 \times 30 \mathrm{~mL}$ methylene dichloride. The combined organic layer was washed with 5\% sodium bicarbonate solution, dried using anhydrous sodium sulfate and evaporated to get compound $\mathbf{1 2}$ as an oily layer. The oil was dissolved in $100 \mathrm{~mL}$ of alcoholic $\mathrm{NaOH}$ solution and heated to reflux for $3 \mathrm{~h}$. The reaction mixture was evaporated under vacuum and diluted to $100 \mathrm{~mL}$ of water. The aqueous layer was extracted using $50 \mathrm{~mL}$ methylene dichloride. Upon acidification of the aqueous layer, the precipitated material was filtered (13) and recrystallized using methanol. Yield; 78\%; mp 240 - $41{ }^{\circ} \mathrm{C}$. IR ( $\left.\mathrm{KBr}, \mathrm{cm}^{-1}\right)$ v 2500-3000 (-OH), 1731 (-CO-), 1690 (-CO- of COOH); ${ }^{1} \mathrm{H} \mathrm{NMR}\left(\mathrm{CDCl}_{3}, 300\right.$ $\mathrm{MHz}) \delta 3.43$ (br, 1H, -OH), 4.28 (d, 2H, $\left.-\mathrm{OCH}_{2-}, J=3.3\right), 4.35$ 
$\left(\mathrm{d}, 2 \mathrm{H},-\mathrm{OCH}_{2^{-}}, J=3.0\right), 7.51$ (t, $2 \mathrm{H}, \mathrm{C}_{3}$ of phenyl, $\left.J=7.8\right), 7.64$ $\left(\mathrm{t}, 1 \mathrm{H}, \mathrm{C}_{4}\right.$ of phenyl, $\left.J=7.2\right), 7.79\left(\mathrm{~d}, 2 \mathrm{H}, \mathrm{C}_{2}\right.$ of phenyl, $\left.J=7.2\right)$.

Synthesis of 7-(phenylcarbonyl)-2,3-dihydrothieno[3,4-b] [1,4]dioxine-5-carbonyl chloride (14): A mixture of $0.5 \mathrm{~g}$ $(0.0019 \mathrm{~mol})$ of keto acid (13) and $15 \mathrm{~mL}$ of thionyl chloride were refluxed for $3 \mathrm{~h}$. Excess thionyl chloride was removed by distillation from the reaction mixture. The liquid was extracted with $50 \mathrm{~mL}$ of methylene dichloride and the organic layer was washed with saturated sodium bicarbonate solution (25 $\mathrm{mL}$ ). After drying the organic layer with anhydrous sodium sulphate, the solvent was removed under vacuum to get of the product, 14. This was used for next step without purification.

General procedure for the synthesis of compounds 15a-e. To a clear solution of $0.5 \mathrm{~g}(0.0016 \mathrm{~mol})$ of acid chloride (14) in methylene dichloride $(10 \mathrm{~mL})$ containing $0.1 \mathrm{~mL}$ of pyridine as a catalyst, equimolar quantity of respective amine were added with stirring. The stirring was continued for $12 \mathrm{~h}$ at $25^{\circ} \mathrm{C}$. The reaction mass was evaporated to dryness and diluted with 100 $\mathrm{mL}$ of water. The product was separated by filtration and recrystallized from appropriate solvent. The physical and characterization data of compounds (15a-e) are given in Table 1. The spectral data of some of the synthesized compounds are as follows:

$\mathrm{N}$-(2,6-Dimethylphenyl)-7-(phenylcarbonyl)-2,3-dihydrothieno[3,4-b][1,4]dioxine-5-carboxamide (15b): $\mathrm{IR}\left(\mathrm{KBr}, \mathrm{cm}^{-1}\right)$ v 3367 (-NH-), 1658 (-CO-), 1626 (-CONH-); ${ }^{1} \mathrm{H} \mathrm{NMR}\left(\mathrm{CDCl}_{3}\right.$, $300 \mathrm{MHz}) \delta 2.28\left(\mathrm{~s}, 6 \mathrm{H},-\mathrm{CH}_{3}\right), 4.37$ (s, $\left.2 \mathrm{H},-\mathrm{OCH}_{2-}, J=3.3\right)$, $4.51\left(\mathrm{~s}, 2 \mathrm{H},-\mathrm{OCH}_{2-}^{-}, J=3.6\right), 7.10$ (s, $3 \mathrm{H}$, xylenyl), $7.45(\mathrm{~m}, 3 \mathrm{H}$, $\mathrm{C}_{3}, \mathrm{C}_{4} \& \mathrm{C}_{5}$ of phenyl), $7.84\left(\mathrm{~d}, 2 \mathrm{H}, \mathrm{C}_{2} \& \mathrm{C}_{6}\right.$ of phenyl, $\left.J=7.2\right)$, 8.19 (s, $1 \mathrm{H},-\mathrm{NH}-) ;{ }^{13} \mathrm{C} \mathrm{NMR}\left(\mathrm{CDCl}_{3}, 75 \mathrm{MHz}\right) \delta 18.5\left(-\mathrm{CH}_{3}\right)$, 64.4-65.2 $\left(-\mathrm{OCH}_{2}-\right), 120.1\left(\mathrm{C}_{3}\right.$ of thiophene $), 120.3\left(\mathrm{C}_{1}\right.$ of xylenyl), 127.4 ( $\mathrm{C}_{3}$ of phenyl $), 128.1\left(\mathrm{C}_{2} \& \mathrm{C}_{6}\right.$ of xylenyl $), 128.9\left(\mathrm{C}_{2}\right.$ of phenyl), 132.5 ( $\mathrm{C}_{4}$ of phenyl), 133.2 ( $\mathrm{C}_{4}$ of thiophene), 135.3 $\left(\mathrm{C}_{3}, \mathrm{C}_{4} \& \mathrm{C}_{5}\right.$ of xylenyl), 138.1 ( $\mathrm{C}_{1}$ of phenyl $), 140.2\left(\mathrm{C}_{5}\right.$ of thiophene), 143.5 ( $\mathrm{C}_{2}$ of thiophene), 158.4 (-CONH-), 187.1 (-CO-); MS (FAB) (m/z, \%): 394 (M+1, 80), 385 (50), 325 (80), 281 (50), 207 (70), 191 (50), 147 (100).

$\mathrm{N}$-(2,3-Dihydro-1H-benzthiazol-2-yl)-2,3-dihydrothieno [3,4-b][1,4] dioxine-5-carboxamide (15d): $\mathrm{IR}\left(\mathrm{KBr}, \mathrm{cm}^{-1}\right) \mathrm{v}$

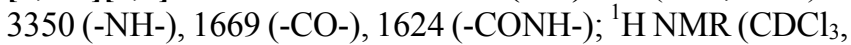
$300 \mathrm{MHz}) \delta 4.41\left(\mathrm{~d}, 2 \mathrm{H},-\mathrm{OCH}_{2-}, J=3.6\right), 4.58\left(\mathrm{~d}, 2 \mathrm{H},-\mathrm{OCH}_{2-}\right.$, $J=3.9$ ), 7.19-7.87 (m, 9H, benzthiazole and phenyl), 10.13 (br, $1 \mathrm{H},-\mathrm{CONH}-$ ).

Pharmacology. Male albino mice (CF-1 strain, 18-25 g) were used for experimentation. The animals were housed in metabolic cages and allowed free access to food and water. The synthesized compounds were suspended in $0.5 \%$ methyl cellulose/water mixture or in polyethylene glycol (PEG 200) and injected intraperitoneally into mice and evaluated in the MES, scMET and neurotoxicity screens. The substances were administered at doses of 30, 100 and $300 \mathrm{mg} / \mathrm{kg}$ at two time intervals.

Maximal Electroshock Test (MES): The MES ${ }^{29-31}$ is a model for generalized tonic-clonic seizures and it provides an indication of ability of a compound to prevent seizure spread when all neuronal circuits in the brain are maximally active. These seizures are highly reproducible and are electrophysiological consistent with human seizures.

For all tests based on MES convulsions, $60 \mathrm{~Hz}$ of alternating current ( $50 \mathrm{~mA})$ was delivered for $2 \mathrm{~s}$ by corneal electrodes, which were primed with an electrolyte solution containing an anesthetic agent $(0.5 \%$ tetracaine $\mathrm{HCl})$. For Test 1 , mice were tested at various intervals following doses of 30, 100 and 300 $\mathrm{mg} / \mathrm{kg}$ of test compound given by i.p. injection of a volume of $0.01 \mathrm{~mL} / \mathrm{g}$. In Test 2 , mice were tested after a dose of $30 \mathrm{mg} / \mathrm{kg}$ (p.o) in a volume of $0.04 \mathrm{~mL} / \mathrm{g}$. Test 3 used varying doses administered via i.p. injection, again in a volume of $0.04 \mathrm{~mL} / \mathrm{g}$. An animal was considered "protected" from MES-induced seizures upon abolition of the hind limb tonic extensor component of the seizure.

Subcutaneous Metrazol Seizure Threshold Test (scMET): This is one of the commonly used tests to measure the ability of a compound to control seizures produced from subcutaneous injection of the metrazol ${ }^{32}$ in mice.

Animals were pretreated with various doses of the test compound (in a similar manner to the MES test, although a dose of $50 \mathrm{mg} / \mathrm{kg}$ (p.o.) was the standard for Test $2 \mathrm{scMET}$ ). At the previously determined TPE of the test compound, the dose of Metrazol which would induce convulsions in $97 \%$ of animals (CD $97: 85 \mathrm{mg} / \mathrm{kg}$ mice) was injected into a loose fold of skin in the midline of the neck. The animals were placed in isolation cages to minimize stress and observed for the next 30 minutes to see the absence of a seizure. An episode of clonic spasms, approximately 3 - 5 seconds, of the fore and/or hind limbs, jaws or vibrissae was taken as the endpoint. Animals, which do not meet this criterion were considered protected.

Neurotoxicity-Minimal Motor Impaiment (MMI): Rotorod technique $^{33}$ is the most widely used method to determine the acute toxicity of compounds in anticonvulsant studies.

In experimental procedure, the mouse was placed on a rod that rotates at a speed of $6 \mathrm{rpm}$, where the animal can maintain its equilibrium for long periods of time. The animal was considered toxic if it falls off this rotating rod three times during a 1-min period. Similar procedure was followed for all the compounds to evaluate neurotoxicity.

Minimal Clonic Seizure (6 Hz) Test: Some clinically useful AEDs are ineffective in the standard MES and scMET tests but still have anticonvulsant activities in vivo. In order to identify potential AEDs with this profile, some compounds were tested in the minimal clonic seizure (6 Hz or psychomotor) test. Like the maximal electroshock (MES) test, the minimal clonic seizure $(6 \mathrm{~Hz})$ test $^{34-35}$ was used to assess compound's efficacy against electrically induced seizures but used a lower frequency $(6 \mathrm{~Hz})$ and longer duration of stimulation $(3 \mathrm{~s})$.

Test compounds were pre-administered to mice via i.p. injection. At varying times, individual mice (four mice per time point) were challenged with sufficient current delivered through corneal electrodes to elicit a psychomotor seizure in $97 \%$ of animals (32 mA for $3 \mathrm{~s}$ ). Untreated mice would display seizures characterized by a minimal clonic phase followed by stereotyped, automatistic behaviors described originally as being similar to the aura of human patients with partial seizures. Animals not displaying this behavior were considered protected.

\section{Results and Discussion}

The scheme 1 describes the synthesis of target molecules. 


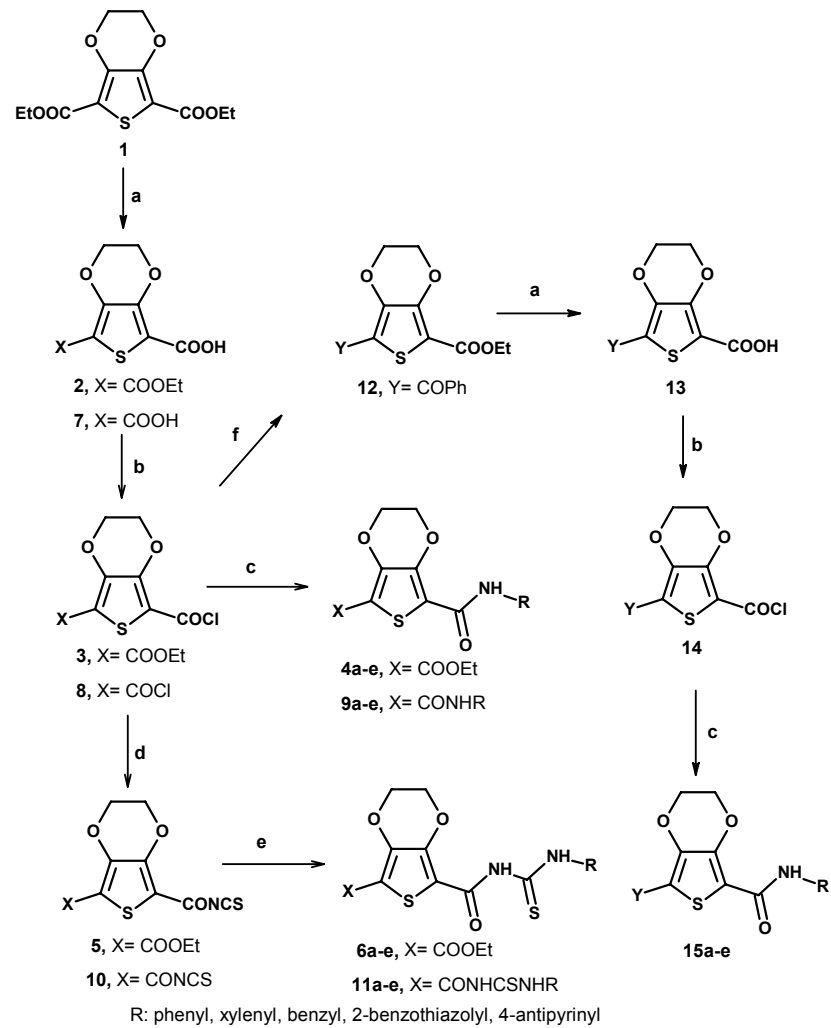

a: ethanolic $\mathrm{NaOH} / \mathrm{H}^{+}$, b: $\mathrm{SOCl}_{2}$, reflux, c: methylenedichloride, amine, pyridine, d: $\mathrm{NH}_{4} \mathrm{SCN}$, methylenedichloride, PEG-600, e: amine, $\mathbf{f}$ $\mathrm{AlCl}_{3}$, benzene.

Scheme 1. Synthesis of thiophene amide and its derivatives

The intermediate monocarboxylic acid $\mathbf{2}$ was synthesized by controlled hydrolysis of ethylenedioxythiophene diester 1 using ethanolic $\mathrm{NaOH}$, whereas the diacid 7 was prepared following the reported procedure. ${ }^{28}$ The acids 2 and 7 were then converted into corresponding acid chlorides 3 and $\mathbf{8}$ by refluxing them with excess thionyl chloride. The final compounds 4a-e and 9a-e were synthesized by treating them ( 3 and $\mathbf{8})$ with corresponding amine in the presence of a base.

In another route, the acid chlorides ( $\mathbf{3}$ and $\mathbf{8}$ ) were treated with ammonium thiocyanate in the presence of phase transfer catalyst, PEG 600 in order to obtain the intermediates, $\mathbf{5}$ and $\mathbf{1 0 .}$ They were conveniently converted into final compounds 6a-e and 11a-e by condensing with different amines.

Further, compound $\mathbf{3}$ was subjected to Friedel Craft's reaction, followed by alkaline hydrolysis to get a carboxylic acid 13 as a solid. This acid was converted into acid chloride 14 by treating with excess thionyl chloride and the final compounds 15a-e were obtained by treating the acid chloride 14 with corresponding amine in the basic medium.

The structural assignments to new compounds were based on their elemental analysis and spectral (FTIR, ${ }^{1} \mathrm{H}$ and ${ }^{13} \mathrm{C}$ NMR and Mass) data. The physical and characterization data of all the newly synthesized compounds are summarized in Table 1.

The IR spectrum of compound $\mathbf{4 b}$ showed the amide -NHpeak at $3367 \mathrm{~cm}^{-1}$. The carbonyl peaks of amide and ester appeared at 1655 and $1720 \mathrm{~cm}^{-1}$ respectively. Further, its ${ }^{1} \mathrm{H}$ NMR spectrum showed a singlet at $\delta 2.27$, which corresponds to methyl group of xylenyl, whereas the amide -NH- group resonated as a singlet at $8.15 \mathrm{ppm}$. The structure of the product was further confirmed by ${ }^{13} \mathrm{C}$ NMR spectral study. The spectrum showed peaks at 158.4 and 160.8 ppm due to the carbonyl carbon of amide and ester respectively. Finally the product was conformed by its mass spectrum. It displayed the molecular ion peak at $361(\mathrm{M}+1)$, which matches with its molecular formula $\mathrm{C}_{18} \mathrm{H}_{19} \mathrm{NO}_{5} \mathrm{~S}$.

The IR spectrum of thioamide $\mathbf{6 b}$ showed the absorption band at $3365 \mathrm{~cm}^{-1}$, due to -NH- stretching of amide group. Further, the carbonyl stretching of amide and ester groups appeared at 1668 and $1716 \mathrm{~cm}^{-1}$ respectively. The -CS- group of thioamide showed the band at $1159 \mathrm{~cm}^{-1}$. The ${ }^{1} \mathrm{H}$ NMR spectrum of the compound $\mathbf{6 b}$ showed sharp singlet peaks at $\delta 4.46$ and 4.55 due to the presence of ethylenedioxy group in the molecule and the ester $-\mathrm{OCH}_{2}$ - group resonated as quartet at 4.37 ppm. The two singlets observed at $\delta 9.96$ and 11.57 were due to -NHCS- and -NHCO- protons of thioamide. In ${ }^{13} \mathrm{C}$ NMR spectrum, it showed signals at $\delta 14.1$ and $\delta 61.6$, which were due to $-\mathrm{CH}_{3}$ and $-\mathrm{OCH}_{2}$ - groups of ester and also it displayed peaks at 64.5 and $65.7 \mathrm{ppm}$, which were due to $-\mathrm{OCH}_{2} \mathrm{CH}_{2} \mathrm{O}$ - of ethylenedioxy thiophene. Also, the amide carbonyl carbon resonated at $159.8 \mathrm{ppm}$ whereas thioamide and ester carbonyl groups appeared at 179.4 and $160.2 \mathrm{ppm}$ respectively. Formation of the compound $\mathbf{6 b}$ from its precursor was further confirmed by its mass spectrum. In its mass spectrum, the compound displayed molecular ion peak at $m / z 421(\mathrm{M}+1,100 \%)$, which is in agreement with the molecular formula, $\mathrm{C}_{19} \mathrm{H}_{20} \mathrm{~N}_{2} \mathrm{O}_{5} \mathrm{~S}_{2}$. The other compounds in this series also followed the same type of fragmentation pattern in their mass spectra.

The IR spectrum of compound 9a showed the peaks at 3381 and $1666 \mathrm{~cm}^{-1}$, which correspond to -NH- and -CO-groups of amide respectively. In ${ }^{1} \mathrm{H}$ NMR spectrum, it displayed a singlet at $9.24 \mathrm{ppm}$, which corresponds to -NH- of amide group. The protons of ethylenedioxy group resonated as a singlet at 4.59 ppm. In its mass spectrum, the compound displayed molecular ion peak at $m / z 381(\mathrm{M}+1,100 \%)$, which is in agreement with the molecular formula, $\mathrm{C}_{20} \mathrm{H}_{16} \mathrm{~N}_{2} \mathrm{O}_{4} \mathrm{~S}$.

The formation of bisthioamide $\mathbf{1 1 b}$ was conformed by its IR. In that, peaks at 3359,1661 and $1150 \mathrm{~cm}^{-1}$ correspond to the presence of -NH-, -CO- and -CS- groups respectively. It was observed that protons of -NHCO- and -NHCS- resonated at 9.9 and $11.5 \mathrm{ppm}$ respectively in its NMR spectrum. In its ${ }^{13} \mathrm{C} \mathrm{NMR}$ spectrum, the carbon of -CO- and -CS- groups resonated at 159.2 and $179.2 \mathrm{ppm}$ respectively. The mass spectrum of it displayed molecular ion peak at $m / z 555(\mathrm{M}+1,100 \%)$, which is in agreement with the molecular formula, $\mathrm{C}_{26} \mathrm{H}_{26} \mathrm{~N}_{4} \mathrm{O}_{4} \mathrm{~S}_{3}$.

The IR spectrum of compound 15a showed absorption bands at 3367,1658 and $1626 \mathrm{~cm}^{-1}$ confirming the presence of amide and benzoyl groups in the molecule. The formation of compound 15a was confirmed by its NMR spectral studies. Its ${ }^{1} \mathrm{H}$ NMR spectrum showed a peak at $\delta 2.28$, which corresponds to $-\mathrm{CH}_{3}$ group of xylenyl and the amide -NH- group, resonated at 8.19 ppm as a singlet. Its ${ }^{13} \mathrm{C}$ NMR spectrum showed the peaks at 158.4 and $187.1 \mathrm{ppm}$ indicating the presence of amide and carbonyl groups in the structure. Further, the mass spectrum of it showed the molecular ion peak at $m / z 394(\mathrm{M}+1,80 \%)$, which agrees with its molecular formula, $\mathrm{C}_{22} \mathrm{H}_{19} \mathrm{NO}_{4} \mathrm{~S}$. 
Table 2. Results of anticonvulsant activity and neurotoxicity of newly synthesized compounds

\begin{tabular}{|c|c|c|c|c|c|c|c|c|c|c|c|c|c|c|c|}
\hline \multirow{2}{*}{ Cpd } & \multirow{2}{*}{$\begin{array}{c}\text { Dose } \\
(\mathrm{mg} / \mathrm{kg})\end{array}$} & \multicolumn{2}{|c|}{$\operatorname{MES}^{a}$} & \multicolumn{2}{|c|}{$\mathrm{scMET}^{b}$} & \multicolumn{2}{|c|}{ Toxicity $^{c}$} & \multirow{2}{*}{ Cpd } & \multirow{2}{*}{$\begin{array}{c}\text { Dose } \\
(\mathrm{mg} / \mathrm{kg})\end{array}$} & \multicolumn{2}{|c|}{$\operatorname{MES}^{a}$} & \multicolumn{2}{|c|}{$\mathrm{scMET}^{b}$} & \multicolumn{2}{|c|}{ Toxicity $^{c}$} \\
\hline & & $0.5 \mathrm{~h}$ & $4 \mathrm{~h}$ & $0.5 \mathrm{~h}$ & $4 \mathrm{~h}$ & $0.5 \mathrm{~h}$ & $4 \mathrm{~h}$ & & & $0.5 \mathrm{~h}$ & $4 \mathrm{~h}$ & $0.5 \mathrm{~h}$ & $4 \mathrm{~h}$ & $0.5 \mathrm{~h}$ & $4 \mathrm{~h}$ \\
\hline \multirow{3}{*}{$4 a$} & 30 & $0 / 1$ & $0 / 1$ & $0 / 1$ & $0 / 1$ & $0 / 4$ & $0 / 2$ & \multirow{3}{*}{ 9d } & 30 & $0 / 1$ & $0 / 1$ & $0 / 1$ & $0 / 1$ & $0 / 4$ & $0 / 2$ \\
\hline & 100 & $0 / 3$ & $0 / 3$ & $0 / 1$ & $0 / 1$ & $0 / 8$ & $0 / 4$ & & 100 & $0 / 3$ & $0 / 3$ & $0 / 1$ & $0 / 1$ & $0 / 8$ & $0 / 4$ \\
\hline & 300 & $0 / 1$ & $0 / 1$ & $0 / 1$ & $0 / 1$ & $0 / 4$ & $0 / 2$ & & 300 & $0 / 1$ & $0 / 1$ & $0 / 1$ & $0 / 1$ & $0 / 4$ & $0 / 2$ \\
\hline \multirow{3}{*}{$4 b$} & 30 & $0 / 1$ & $0 / 1$ & $0 / 1$ & $0 / 1$ & $0 / 4$ & $0 / 2$ & \multirow{3}{*}{$9 e$} & 30 & $0 / 1$ & $0 / 1$ & $0 / 1$ & $0 / 1$ & $0 / 4$ & $0 / 2$ \\
\hline & 100 & $0 / 3$ & $0 / 3$ & $0 / 1$ & $0 / 1$ & $0 / 8$ & $0 / 4$ & & 100 & $0 / 3$ & $0 / 3$ & $0 / 1$ & $0 / 1$ & $0 / 8$ & $0 / 4$ \\
\hline & 300 & $0 / 1$ & $0 / 1$ & $0 / 1$ & $0 / 1$ & $0 / 4$ & $0 / 2$ & & 300 & $0 / 1$ & $0 / 1$ & $0 / 1$ & $0 / 1$ & $0 / 4$ & $0 / 2$ \\
\hline \multirow{3}{*}{$4 c$} & 30 & $0 / 1$ & $0 / 1$ & $0 / 1$ & $0 / 1$ & $0 / 4$ & $0 / 2$ & \multirow{3}{*}{$11 \mathrm{a}$} & 30 & $0 / 1$ & $0 / 1$ & $0 / 1$ & $0 / 1$ & $0 / 4$ & $0 / 2$ \\
\hline & 100 & $0 / 3$ & $0 / 3$ & $0 / 1$ & $0 / 1$ & $0 / 8$ & $0 / 4$ & & 100 & $0 / 3$ & $0 / 3$ & $0 / 1$ & $0 / 1$ & $0 / 8$ & $0 / 4$ \\
\hline & 300 & $0 / 1$ & $0 / 1$ & $0 / 1$ & $0 / 1$ & $0 / 4$ & $0 / 2$ & & 300 & $0 / 1$ & $0 / 1$ & $0 / 1$ & $0 / 1$ & $0 / 4$ & $0 / 2$ \\
\hline \multirow{3}{*}{$4 d$} & 30 & $0 / 1$ & $0 / 1$ & $0 / 1$ & $0 / 1$ & $0 / 4$ & $0 / 2$ & \multirow{3}{*}{$11 b$} & 30 & $0 / 1$ & $0 / 1$ & $0 / 1$ & $0 / 1$ & $0 / 4$ & $0 / 2$ \\
\hline & 100 & $0 / 3$ & $0 / 3$ & $0 / 1$ & $0 / 1$ & $0 / 8$ & $0 / 4$ & & 100 & $0 / 3$ & $0 / 3$ & $0 / 1$ & $0 / 1$ & $0 / 8$ & $0 / 4$ \\
\hline & 300 & $0 / 1$ & $0 / 1$ & $0 / 1$ & $0 / 1$ & $0 / 4$ & $0 / 2$ & & 300 & $0 / 1$ & $0 / 1$ & $0 / 1$ & $0 / 1$ & $0 / 4$ & $0 / 2$ \\
\hline \multirow{3}{*}{$4 e$} & 30 & $0 / 1$ & $0 / 1$ & $0 / 1$ & $0 / 1$ & $0 / 4$ & $0 / 2$ & \multirow{3}{*}{$11 \mathrm{c}$} & 30 & $0 / 1$ & $0 / 1$ & $0 / 1$ & $0 / 1$ & $0 / 4$ & $0 / 2$ \\
\hline & 100 & $0 / 3$ & $0 / 3$ & $0 / 1$ & $0 / 1$ & $0 / 8$ & $0 / 4$ & & 100 & $0 / 3$ & $0 / 3$ & $0 / 1$ & $0 / 1$ & $0 / 8$ & $0 / 4$ \\
\hline & 300 & $0 / 1$ & $0 / 1$ & $0 / 1$ & $0 / 1$ & $0 / 4$ & $0 / 2$ & & 300 & $0 / 1$ & $0 / 1$ & $0 / 1$ & $0 / 1$ & $0 / 4$ & $0 / 2$ \\
\hline \multirow{3}{*}{$\mathbf{6 a}$} & 30 & $0 / 1$ & $0 / 1$ & $0 / 1$ & $0 / 1$ & $0 / 4$ & $0 / 2$ & & 30 & $0 / 1$ & $0 / 1$ & $0 / 1$ & $0 / 1$ & $0 / 4$ & $0 / 2$ \\
\hline & 100 & $0 / 3$ & $0 / 3$ & $0 / 1$ & $0 / 1$ & $0 / 8$ & $0 / 4$ & $11 d$ & 100 & $0 / 3$ & $0 / 3$ & $0 / 1$ & $0 / 1$ & $0 / 8$ & $0 / 4$ \\
\hline & 300 & $0 / 1$ & $0 / 1$ & $0 / 1$ & $0 / 1$ & $0 / 4$ & $0 / 2$ & & 300 & $0 / 1$ & $0 / 1$ & $0 / 1$ & $0 / 1$ & $0 / 4$ & $0 / 2$ \\
\hline & 30 & $0 / 1$ & $0 / 1$ & $0 / 1$ & $0 / 1$ & $0 / 4$ & $0 / 2$ & & 30 & $0 / 1$ & $0 / 1$ & $0 / 1$ & $0 / 1$ & $0 / 4$ & $0 / 2$ \\
\hline $6 b$ & 100 & $0 / 3$ & $0 / 3$ & $0 / 1$ & $0 / 1$ & $0 / 8$ & $0 / 4$ & $11 \mathrm{e}$ & 100 & $0 / 3$ & $0 / 3$ & $0 / 1$ & $0 / 1$ & $0 / 8$ & $0 / 4$ \\
\hline & 300 & $0 / 1$ & $0 / 1$ & $0 / 1$ & $0 / 1$ & $0 / 4$ & $0 / 2$ & & 300 & $0 / 1$ & $0 / 1$ & $0 / 1$ & $0 / 1$ & $0 / 4$ & $0 / 2$ \\
\hline & 30 & $0 / 1$ & $0 / 1$ & $0 / 1$ & $0 / 1$ & $0 / 4$ & $0 / 2$ & & 30 & $0 / 1$ & $0 / 1$ & $0 / 1$ & $0 / 1$ & $0 / 4$ & $0 / 2$ \\
\hline $6 c$ & 100 & $0 / 3$ & $0 / 3$ & $0 / 1$ & $0 / 1$ & $0 / 8$ & $0 / 4$ & $15 a$ & 100 & $0 / 3$ & $0 / 3$ & $0 / 1$ & $0 / 1$ & $0 / 8$ & $0 / 4$ \\
\hline & 300 & $0 / 1$ & $0 / 1$ & $0 / 1$ & $0 / 1$ & $0 / 4$ & $0 / 2$ & & 300 & $0 / 1$ & $0 / 1$ & $0 / 1$ & $0 / 1$ & $0 / 4$ & $0 / 2$ \\
\hline & 30 & $0 / 1$ & $0 / 1$ & $0 / 1$ & $0 / 1$ & $0 / 4$ & $0 / 2$ & & 30 & $0 / 1$ & $0 / 1$ & $0 / 1$ & $0 / 1$ & $0 / 4$ & $0 / 2$ \\
\hline $6 d$ & 100 & $0 / 3$ & $0 / 3$ & $0 / 1$ & $0 / 1$ & $0 / 8$ & $0 / 4$ & $15 b$ & 100 & $0 / 3$ & $0 / 3$ & $0 / 1$ & $0 / 1$ & $0 / 8$ & $0 / 4$ \\
\hline & 300 & $0 / 1$ & $0 / 1$ & $0 / 1$ & $0 / 1$ & $0 / 4$ & $0 / 2$ & & 300 & $0 / 1$ & $0 / 1$ & $0 / 1$ & $0 / 1$ & $0 / 4$ & $0 / 2$ \\
\hline & 30 & $0 / 1$ & $0 / 1$ & $0 / 1$ & $0 / 1$ & $0 / 4$ & $0 / 2$ & & 30 & $0 / 1$ & $0 / 1$ & $0 / 1$ & $0 / 1$ & $0 / 4$ & $0 / 2$ \\
\hline $6 e$ & 100 & $0 / 3$ & $0 / 3$ & $0 / 1$ & $0 / 1$ & $0 / 8$ & $0 / 4$ & $15 c$ & 100 & $0 / 3$ & $0 / 3$ & $0 / 1$ & $0 / 1$ & $0 / 8$ & $0 / 4$ \\
\hline & 300 & $0 / 1$ & $0 / 1$ & $0 / 1$ & $0 / 1$ & $0 / 4$ & $0 / 2$ & & 300 & $0 / 1$ & $0 / 1$ & $0 / 1$ & $0 / 1$ & $0 / 4$ & $0 / 2$ \\
\hline & 30 & $0 / 1$ & $0 / 1$ & $0 / 1$ & $0 / 1$ & $0 / 4$ & $0 / 2$ & & 30 & $0 / 1$ & $0 / 1$ & $0 / 1$ & $0 / 1$ & $0 / 4$ & $0 / 2$ \\
\hline $9 \mathbf{a}$ & 100 & $0 / 3$ & $0 / 3$ & $0 / 1$ & $0 / 1$ & $0 / 8$ & $0 / 4$ & $15 d$ & 100 & $0 / 3$ & $0 / 3$ & $0 / 1$ & $0 / 1$ & $0 / 8$ & $0 / 4$ \\
\hline & 300 & $0 / 1$ & $0 / 1$ & $0 / 1$ & $0 / 1$ & $0 / 4$ & $0 / 2$ & & 300 & $0 / 1$ & $0 / 1$ & $0 / 1$ & $0 / 1$ & $0 / 4$ & $0 / 2$ \\
\hline & 30 & $0 / 1$ & $0 / 1$ & $0 / 1$ & $0 / 1$ & $0 / 4$ & $0 / 2$ & & 30 & $0 / 1$ & $0 / 1$ & $0 / 1$ & $0 / 1$ & $0 / 4$ & $0 / 2$ \\
\hline $9 b$ & 100 & $0 / 3$ & $0 / 3$ & $0 / 1$ & $0 / 1$ & $0 / 8$ & $0 / 4$ & $15 e$ & 100 & $0 / 3$ & $0 / 3$ & $0 / 1$ & $0 / 1$ & $0 / 8$ & $0 / 4$ \\
\hline & 300 & $0 / 1$ & $0 / 1$ & $0 / 1$ & $0 / 1$ & $0 / 4$ & $0 / 2$ & & 300 & $0 / 1$ & $0 / 1$ & $0 / 1$ & $0 / 1$ & $0 / 4$ & $0 / 2$ \\
\hline & 30 & $0 / 1$ & $0 / 1$ & $0 / 1$ & $0 / 1$ & $0 / 4$ & $0 / 2$ & & & & & & & & \\
\hline $9 c$ & 100 & $0 / 3$ & $0 / 3$ & $0 / 1$ & $0 / 1$ & $0 / 8$ & $0 / 4$ & & & & & & & & \\
\hline & 300 & $0 / 1$ & $0 / 1$ & $0 / 1$ & $0 / 1$ & $0 / 4$ & $0 / 2$ & & & & & & & & \\
\hline
\end{tabular}

${ }^{a}$ Maximal Electroshock test. ${ }^{b}$ Subcutaneous Metrazol Seizure Threshold test. ${ }^{c}$ Rotorod toxicity.

Anticonvulsant activity. The newly synthesized compounds were evaluated for anticonvulsant activity by the maximal electroshock (MES), subcutaneous metrazol (scMET) and neurotoxicity screening by Rotorod technique. Some of the selected compounds were also evaluated in $6 \mathrm{~Hz}$ model at five different time intervals. The results of anticonvulsant activity of the newly synthesized compounds 4(a-e), 6(a-e), 9(a-e), 11(a-e) and 15 (a-e) are presented in Tables 2 and 3. The anticonvulsant evaluations were carried out by the National Institute of Health, National Institute of Neurological Disorders and Strokes (NINDS), USA, following reported procedures.

The neurotoxicity results of the newly synthesized compounds indicated that they were nontoxic at a maximum dose of 300 $\mathrm{mg} / \mathrm{kg}$ body weight. In our earlier article ${ }^{27}$ we reported that the toxicity of thiophene bis hydrazones was attributed to the presence of secondary aryl group but not due to the presence of 3,4 -disubstituted thiophene nucleus in the structure. Here, the results of neurotoxicity screening of target molecules clearly supported the results of our previous work. However, the results of MES and scMET screening indicated that the newly synthesized compounds were not active at a maximum dose of $300 \mathrm{mg} / \mathrm{kg}$ at the time point of evaluation. Hence, some of the compounds were selected for $6 \mathrm{~Hz}$ testing to evaluate their uncover activity from the traditional MES screening. Their results revealed that the compounds $4 \mathrm{~b}, \mathbf{6 a}, 6 \mathrm{~b}, \mathbf{6 d}, 9 \mathrm{~b}, \mathbf{1 1 c}, \mathbf{1 5 a}$ and $15 \mathbf{b}$ showed activity at $100 \mathrm{mg} / \mathrm{kg}$ of body weight. 
Table 3. Results of anticonvulsant activity by $6 \mathrm{~Hz}$ model

\begin{tabular}{ccccccc}
\hline Cpd & $\begin{array}{c}\text { Dose } \\
(\mathrm{mg} / \mathrm{kg})\end{array}$ & $0.25 \mathrm{~h}$ & $0.5 \mathrm{~h}$ & $1.0 \mathrm{~h}$ & $2.0 \mathrm{~h}$ & $4.0 \mathrm{~h}$ \\
\hline 4b & 100 & $0 / 4$ & $0 / 4$ & $2 / 4$ & $0 / 4$ & $0 / 4$ \\
$\mathbf{4 d}$ & 100 & $0 / 4$ & $0 / 4$ & $0 / 4$ & $0 / 4$ & $0 / 4$ \\
$\mathbf{4 c}$ & 100 & $0 / 4$ & $0 / 4$ & $0 / 4$ & $0 / 4$ & $0 / 4$ \\
$\mathbf{6 a}$ & 100 & $0 / 4$ & $3 / 4$ & $1 / 4$ & $1 / 4$ & $0 / 4$ \\
$\mathbf{6 b}$ & 100 & $1 / 4$ & $1 / 4$ & $2 / 4$ & $2 / 4$ & $0 / 4$ \\
$\mathbf{6 d}$ & 100 & $1 / 4$ & $2 / 4$ & $1 / 4$ & $1 / 4$ & $0 / 4$ \\
9b & 100 & $1 / 4$ & $0 / 4$ & $2 / 4$ & $1 / 4$ & $0 / 4$ \\
9c & 100 & $0 / 4$ & $1 / 4$ & $1 / 4$ & $1 / 4$ & $0 / 4$ \\
$\mathbf{1 1 a}$ & 100 & $1 / 4$ & $0 / 4$ & $1 / 4$ & $1 / 4$ & $0 / 4$ \\
$\mathbf{1 1 c}$ & 100 & $0 / 4$ & $2 / 4$ & $0 / 4$ & $1 / 4$ & $0 / 4$ \\
$\mathbf{1 1 d}$ & 100 & $0 / 4$ & $1 / 4$ & $0 / 4$ & $0 / 4$ & $0 / 4$ \\
$\mathbf{1 5 a}$ & 100 & $1 / 4$ & $2 / 4$ & $1 / 4$ & $0 / 4$ & $0 / 4$ \\
$\mathbf{1 5 b}$ & 100 & $0 / 4$ & $1 / 4$ & $2 / 4$ & $0 / 4$ & $0 / 4$ \\
\hline
\end{tabular}

Amongst the tested compounds in amide series, the compound $\mathbf{4 b}$, containing xylenyl pharmacophroe attached to amide group showed $50 \%$ production at one hour time period. But, the compound $\mathbf{4 c}$ which contains benzyl group in the place of xylenyl group did not show any activity. Further, it was observed that when the ester group was replaced by benzoyl moiety, the activity was retained. In this series the compounds $\mathbf{1 5 a}$ and $\mathbf{1 5 b}$ which contain phenyl and xylenyl groups linked to amide, respectively showed good results. It is interesting to note that amongst the tested compounds in bisamide series, the compound 9b carrying xylenyl group showed better resistance than the other compounds.

In the thioamide series, the compounds $\mathbf{6 a}, \mathbf{6 b}$ and $\mathbf{6 d}$ containing aniline, xylenyl and 2-aminobenzothiazole moieties respectively showed better protection than the corresponding amide series. It was noticed that introduction of methyl group in the phenyl ring brought about enhanced duration of the action against induced convulsion. But the resistivity of the compound 6b reduced to $50 \%$ when compared to that of the compound 6a. Similarly, in the case of compound $\mathbf{6 d}$, the activity was reduced to $50 \%$ when the phenyl group was replaced by benzothiazole ring. But, the duration of its action was reduced when compared to that of the compound $\mathbf{6 b}$. The results of $6 \mathrm{~Hz}$ screening clearly indicated that the compound $\mathbf{6 a}$ emerged as better candidate than the other compounds in this series. In the bisthioamide series, the compound 11c that contains benzyl group showed positive response against induced convulsions. It was observed that the presence of ester pharmacophore in the molecule caused increased results.

\section{Conclusion}

The present study highlights the design, synthesis and characterization of the newly synthesized five series of 3,4-ethylenedioxythiophene derivatives and evaluation of their anticonvulsant activity by different models along with their neurotoxicity. It was noticed that in the amide (4a-e) and bisamide (6a-e) series, xylenyl pharmacophore showed better results than the other groups whereas in the thioamide (9a-e) series, the presence of ester functional group enhanced the activity con- siderably. Further, presence of aniline and xylenyl moieties in the compounds 9a-e and 11a-e caused increased activity. From the study it can be concluded that compounds $\mathbf{6 a}, \mathbf{b}, \mathbf{d}$ emerged as potential leads for further structural modifications.

Out of five series, the thioamide series were found to be more active than the remaining series. Further, the anticonvulsant study clearly showed that the presence of the pharmacophoric elements such as ether linkage, ester, thiophene and amide/ thioamide groups has influenced the activity to some extent. Also, the toxicity study revealed that the different substituents at positions-2 and -5 of 3,4-ethylenedioxythiophene have not affected the results.

Acknowledgments. The authors are thankful to the Head, SAIF, CDRI Lucknow and the Chairman, NMRRC, IISc, Bangalore for providing mass, ${ }^{1} \mathrm{H}$ NMR and ${ }^{13} \mathrm{C}$ NMR spectral data.

\section{References}

1. Somjen, G. G. Ions in the brain: Normal function, Seizures and Stroke; Oxford University press: New York, 2004; pp 107-240.

2. Flaherty, P. T.; Greenwood, T. D.; Manheim, A. L,; Wolf, J. F. J. Med. Chem. 1996, 39, 1509.

3. John, B. H.; Anders, F. J.; Birgitte, V. C.; Frederick, C. G.; Lone, J.; John, P. M.; Erik, B. N.; Mark, A. S.; Francis, J. W.; Xu-Feng, Z. Eur. J. Med. Chem. 1998, 33, 839.

4. Maria, L. B.; Gabriela, M. M.; Yolanda, K. C.; Raquel de Oliveira, L.; Everton, T.; Aline, C.; Salete, S.; Magna, S. A.; Eliezer, J. B.; Lidia, M. L. Eur. J. Med. Chem. 2009, 44, 3612.

5. Diouf, O.; Bourhim, M.; Lambert, D. M.; Poupaert, J. H.; Stables, J. P.; Vamecq, J. Biomed. Pharmacother. 1997, 51, 131.

6. Micheal, P. K.; Jacop, S. US patent 4186208, 1980.

7. Zeynep, A.; Fatma, K.; Kevser, E.; Varol, P. Il Farmaco. 2002, 57,201

8. Cecile, B.; Arnaud, L.; Stables, J. P.; Robert, D. V.; Harold, K. Bioorg. Med. Chem. 2004, 12, 3079.

9. Clark, C. R. Epilepsia. 1988, 29, 198.

10. Bettina, K. B.; Joachim, F.; Cara, H.; Niels, K.; Ute, S.; Thomas, S. CNS Drug Reviews 2007, 13, 21.

11. Lepage, F.; Tombret, F.; Cuvier, G.; Marivain, A.; Gillardin, J. M. Eur. J. Med. Chem. 1992, 27, 581.

12. Barbara, M.; Katarzyna, K.; Agnieszka, S.; Stables, J. P. Bioorg. Med. Chem. 2004, 12, 635.

13. Cecile, B.; Shridhar, V. A.; Albert, Y. J.; Stables, J. P.; Donald, F. W.; Harold, K. Bioorg. Med. Chem. 2003, 11, 275.

14. Du, J.; Suzuki, K.; Wei, Y.; Wang, Y.; Blumenthal, R.; Chen, Z.; Falke, C.; Zarate, C. A.; Manji, H. K. Neuropsychopharmacol. 2007, 32, 793 .

15. Yogeeswari, P.; Sriram, P.; Mehta, S.; Nigam. D.; Mohankumar, M.; Murugesan, S.; Stables, J. P. Il Farmaco. 2005, 60, 1.

16. Nadeem, S.; Surendra, N. P.; Suroor, A. K.; Stables, J. P.; Arpana, R.; Mahfuz, A.; Faiz, A. M.; Mashooq, A. B. Bioorg. Med. Chem. Lett. 2007, 17, 255.

17. Bialer, M.; Johannessen, S. I.; Kupferberg, H. J.; Levy, R. H.; Perucca, E.; Tomson, T. Epilepsy Res. 2007, 73, 1.

18. Tomson, T. Curr. Sci. 2002, 86, 698.

19. Nicola, M.; Maria, Z.; Giuseppe, Z.; Frank, S. M.; Guido, F.; Giovambattista De Sarro.; Silvana, G. Il Framaco. 2005, 60, 231.

20. Maria, Z.; Silvan, G.; Nicola, M.; Giuseppe, Z.; Frank, S. M.; Guido, F.; Giovambattista De Sarro.; Carlo De Micheli. Bioorg. Med. Chem. Lett. 2003, 13, 4427.

21. Maria, Z.; Alessia, P.; Nicola, M.; Frank, S.M.; Guido, F.; Giovamattista De Sarro,; Silvana, G.; Carlo De Micheli. Bioorg. Med. Chem. Lett. 2006, 16, 167.

22. Nicola, M.; Giovambattista De Sarro.; Guido, F.; Maria, Z.; Sil- 
vana, G.; Giulia, P.; Carlo De Micheli, Bioorg. Med. Chem. 2004, 12,3703

23. Natalie, D. E.; Donna, S. C.; Manoj, D.; Noha, N. S.; Stables, J. P.; Sylvia, J. H.; Abraham, N.; Robert, S. T.; Uy, Q. T.; Jacqueline, A. M.; Judith, C. B.; Scott, K. R. Eur. J. Med. Chem. 2003, 38, 49.

24. Klaus, U.; Jurgen, E.; Norbert, H.; Angelika, R.; Ralf, G.; HansJoachim, L.; Manfred, M.; Andreas, R.; Jurgen, L.; Birgit, M.; Hans-Jorg, H. J. Med. Chem. 1998, 41, 63.

25. William, J. G. J. Epilepsy. 1994, 7, 161.

26. Polivka, Z.; Holubek, J.; Svatek, E.; Metys, J.; Protiva, M. Coll. Czech. Chem. Commun. 1984, 49, 621.

27. Ravi, K.; Adhikari, A. V.; Stables, J. P. Eur. J. Med. Chem. 2009, 44, 4376.

28. Ojha, U. P.; Krishnamoorthy, K.; Anilkumar, Synth. Met. 2003, $132,279$.

29. Swinyard, E. A.; Woodhead, J. H.; White, H. S.; Franklin, M. R. General Principles: Experimental Selection, Quantification and
Evaluation of Anticonvulsants, in Antiepileptic Drugs; Levy RHM, R. H., Melrum, B., Penry, J. K., Dreifussed, F. E., Eds.; Raven Press: New York, 1989; pp 85-102.

30. White, H. S.; Johnson, M.; Wolf, H. H.; Kupferberg, H. F. Ital J. Nrurol. Sci. 1995a, 16, 73.

31. White, H. S.; Woodhead, J. H.; Franklin, M. R. General Principles: Experimental Selection, Quantification and Evaluation of Antiepileptic Drugs, in Antiepileptic Drugs; Levy RHM, R. H.; Meldrum, B. S., Eds.; Raven Press: New York, 1995; pp 99-110.

32. Swinyard, E. A.; Clark, L. D.; Miyahara, J. T.; Wolf, H. H. J. Physiol. 1961, 132, 97.

33. Dunham, M. S.; Miya, T. A. J. Amer. Pharm. Ass. Sci. Ed. 1957, 46, 208

34. Barton, M. E.; Klein, B. D.; Wolf, H. H.; White, H. S. Epilepsy. Res. 2001, 47, 217.

35. Toman, J. E.; Everett, G. M.; Richards, R. K. Tex. Rep. Biol. Med. 1952, 10, 96 . 